\title{
DESIGNING TWO-CHANNEL \\ CAUSAL STABLE IIR PR FULTER BANKS AND WAVELET BASES BY MODEL ORDER REDUCTION AND CONSTRAINED OPTIMIZATION
}

\author{
J. S. Mao, S. C. Chan and K. L. Ho \\ Department of Electrical and Electronic Engineering \\ The University of Hong Kong, Pokfulam Road, Hong Kong
}

\begin{abstract}
In this paper, two methods for designing two-channel causal stable IIR PR filter banks are introduced. The first method makes use of model reduction and constrained optimization to obtain a causal stable IIR tilter bank from the structural PR FIR filter bank proposed in [2]. It yields better frequency characteristics than the original FIR filter bank and avoids the dump at $\pi / 2$ when allpass filters are used. Using the $1-D$ to $2-D$ transformation proposed in [2], two dimensional PR IIR filter bank can readily be obtained from these prototypes. The second method is based on constrained optimization technique using the general PR condition. Using this technique, filter banks with low system delay and flexible frequency characteristics can be designed. The technique can also be modified to design causal stable IIR dyadic wavelet bases with added regularity conditions. A number of design examples are used to demonstrate the usefulness of the proposed design methods.
\end{abstract}

\section{INTRODUCTION}

Perfect reconstruction (PR) IIR filter banks are very attractive alternative to the conventional FIR systems due to their potentially low system delay and better frequency responses [1]. The design of IIR filter banks, however, is very involved. In addition to the more complicated $P R$ condition, it is also very difficult to ensure that the IIR filters be causal stable. One very good design of PR IIR filter bank is the structural PR system proposed in [2] using all-pass function. There is, however, a 4 $\mathrm{dB}$ dump at $\omega=\pi / 2$ and, because of the PR structure, the attenuation of the analysis lowpass filter is about $10 \mathrm{~dB}$ higher than that of the highpass filter. Method to reduce this problem has recently been proposed in [4], but the stability of the filters cannot be guaranteed.

In this paper, two methods for designing two-channel causal stable IIR PR filter banks are introduced. The first method makes use of model reduction and constrained optimization to obtain a causal stable IIR filter bank from the structural PR FIR filter bank proposed in [2]. It yields better frequency characteristics than the original FIR filter bank and avoids the dump at $\pi / 2$ when allpass filters are used. Using the transformation proposed in [2], two dimensional IIR PR filter bank can readily be obtained from these prototypes. The second method is based on constrained optimization technique using the general PR condition. Using this technique and the model reduced pseudo PR filter bank as initial guess, filter banks with low system delay and flexible frequency characteristics can be designed. Furthermore, these IIR filter banks can be used to obtain dyadic wavelet bases with added regularity conditions $[7,8]$. Design examples show that both of these techniques are very effective in designing two-channel causal stable IIR filter banks. The layout of the paper is as follows: Section 2 is devoted to the first method using model reduction technique. The PR conclition and the constrained optimization method will be described in Section 3. The design of the wavelet bases and the 2D nonseparable filter bank will be discussed in Section 4 and Section 5 , respectively.

\section{DESIGN OF IIR PR FILTER BANKS VIA MODEL REDUCTION}

\subsection{Structural PR filter bank}

A two-channel critical decimated tilter bank is Perfection Reconstruction (PR) if the systen transform function $T(z)$ satisfies

$$
T(z)=\frac{1}{2}\left[H_{\mathrm{s}}(-z) H_{1}(z)-H_{\mathrm{u}}(z) H_{1}(-z)\right]=c z^{-n_{0}},
$$

where $H_{\mathrm{a}}(z)$ and $H_{1}(z)$ are the analysis lowpass and highpass filters, respectively. $n_{*}$ is an integer and $c$ is a non-zero constant. In [2], a class of structural PR two-channel FIR and IIR filter banks were proposed, where the analysis filters are given by,

$$
\begin{aligned}
& H_{\mathrm{a}}(z)=\frac{\left(z^{-2 x}+z^{-1} \beta\left(z^{2}\right)\right)}{2} \\
& H_{1}(z)=-\beta\left(z^{2}\right) H_{n}(z)+z^{-x+1}
\end{aligned}
$$

In the IfR case, $\beta(z)$ is chosen to be a causal stable allpass function. Filter banks with very high stopband attenuation can be designed. Unfortunately, the IIR filter, using the allpass function, has a bump of approximately $4 \mathrm{~dB}$ at $\omega=\pi / 2$, no matter how $\beta(z)$ is designed. In the FIR case, $\beta(z)$ is chosen to be a Type 2 linear phase function with magnitude response as close to unity as possible. Though there are slight dumping at $\omega=\pi / 2$, it is much less serious than the IIR case. If model reduction technique [6] is used to approximate $\beta(z)$ by a causal stable IIR function $\hat{\beta}(z)$, the dumping can be greatly reduced. To further irnprove the stopband attenuation of the filter bank, 
the coefficients of $\hat{\beta}(z)$ can be optimized as the following constrained optimization,

$$
\min _{x} \Phi \text {, subjected to } \hat{\beta}(z) \text { be causal stable. }
$$

Since the filter bank is biorthogonal, both the passband ripples and the stopband attenuation of the analysis filters have to be minimized. A possible objective function is,

$$
\Phi=\left.\int_{0}^{\varepsilon_{0}}|1-| H_{0}\left(e^{j \omega}\right)\right|^{2} d \omega+\int_{\omega_{0}}^{2}\left|H_{0}\left(e^{j \omega}\right)\right|^{2} d \omega,
$$

where, $\omega_{p}$ and $\omega_{s}$ are, respectively, the pass-band and stopband cutoff frequencies. The variables, $X$, are the coefficients of $\hat{\beta}(z)$.

\subsection{Design Example}

In this example, the length of the FIR filter, $\beta(z)$, is $N=12$ [2]. Model order reduction is then used to convert $\beta(z)$ to an IIR filter, $\hat{\beta}(z)$, with numerator and denominator polynomials of order 6 . The NCONF/DCONF subroutine in the IMSL library is used to perform the optimization. The magnitude responses of the original FIR analysis filters $\left(H_{0}(z), H_{1}(z)\right)$ and the optimized

IIR filters $\left(\hat{H}_{0}(z), \hat{H}_{1}(z)\right)$ are shown in Fig. 1 . It can be seen that the optimized system has better frequency characteristic than the original FIR system and there are almost no dumps in the transition band.

\section{DESIGN OF TWO-CHANNEL IR PR FILTER BANK USING CONSTRAINED OPTIMIZATION}

\subsection{PR Condition}

Let the analysis and synthesis filters be given by,

$$
H_{i}(z)=\frac{N_{i}(z)}{D_{i}(z)} \quad i=0,1,
$$

where $N_{i}(z)$ and $D_{i}(z), i=0,1$, are polynomials in $z$. To determine its PR condition, we need to express $H_{0}(z)$ and $H_{1}(z)$ in their polyphase representations. We can multiply the numerator and denominator of $H_{i}(z)$ by $D_{i}(-z)$ to obtain,

$$
H_{i}(z)=\frac{N_{i}(z) D_{i}(-z)}{D_{i}(z) D_{i}(-z)}=\frac{\tilde{N}_{i}(z)}{\tilde{D}_{i}\left(z^{2}\right)} .
$$

Decomposing $\bar{N}_{i}(z)$ into its polyphase components, we have

$$
H_{i}(z)=\frac{\tilde{N}_{i 0}\left(z^{2}\right)}{\tilde{D}_{i}\left(z^{2}\right)}+z^{-1} \frac{\tilde{N}_{i 1}\left(z^{2}\right)}{\tilde{D}_{i}\left(z^{2}\right)} \text {. }
$$

Therefore, the analysis polyphase matrix is given by,

$$
E(z)=\left[\begin{array}{cc}
\frac{\tilde{N}_{00}(z)}{\tilde{D}_{0}(z)} & \frac{\tilde{N}_{01}(z)}{\tilde{D}_{0}(z)} \\
\frac{\tilde{N}_{10}(z)}{\tilde{D}_{1}(z)} & \frac{\tilde{N}_{11}(z)}{\tilde{D}_{1}(z)}
\end{array}\right] .
$$

To achieve PR, the determinant of $E(z)$ should in general be a minimum phase function. In PR FIR filter banks, the minimum phase function will reduce to simple signal delay. In this paper, we shall limit ourselves to the latter case to simplify the PR condition. In this case, we have

$$
\operatorname{det}[E(z)]=\frac{\tilde{N}_{\mathrm{kg}}(z) \tilde{N}_{11}(z)-\tilde{N}_{101}(z) \tilde{N}_{01}(z)}{\tilde{D}_{0}(z) \tilde{D}_{1}(z)}=c \cdot z^{-d}
$$

where $d$ is an integer and $\beta$ is a non-zero constant. Equivalently, we have.

$$
\tilde{N}_{b 11}(z) \tilde{N}_{11}(z)-\tilde{N}_{11}(z) \tilde{N}_{111}(z)=c \cdot z^{-l} \tilde{D}_{b}(z) \tilde{D}_{1}(z)
$$

(3-6) is similar to the FIR case except that the right hand side is now a polynomial. To ensure that the filters are causal stable, the poles of $H_{i}(z)$ should remain inside the unit cycle. In other words, the zeros of $\tilde{D}_{i}(z)$ should be inside the unit cycle. For simplicity of notation, it is assumed that all the zeros of $\tilde{D}_{i}(z)$ occur in complex conjugate pairs so that

$$
\tilde{D}_{i}(z)=K_{i} \prod_{k=1}^{N i}\left(1-p_{i, k} \cdot z^{-1}\right)\left(1-p_{i, k}^{*} \cdot z^{-1}\right) .
$$

Modifications to include a fixed number of real zeros are easily made. The design problem can be formulated as the following constrained optimization problem,

$$
\begin{aligned}
& \min _{x} \Phi(X), \\
& \text { subjected to: }\left|p_{i, k}\right|<1, i=0,1, k=1, \ldots, N D_{i}, \\
& \text { and the PR conditions in }(3-6) .
\end{aligned}
$$

The objective function is the same as $(2-4)$. The variables, $X$, to be optimized are the coefficients of the poiynomials $\tilde{N}_{i j}(\xi)$, $i, j=0,1$, and the real and imaginary parts of $p_{i, k}, i=0,1$; $k=1, \ldots, N D_{i}\left(r_{-} p_{i, k}\right.$ and $\left.i_{-m} p_{i, k}\right)$.

\subsection{Initial Guess and Order Selection}

The convergence and computational time required for the optimization are usually significantly affected by the choice of the initial guess. In designing low order IIR PR filter banks, a PR FIR filter bank with similar characteristic can first be designed and used as the numerator of the initial gucss. The denominators can be chosen as 1 with $p_{i, \alpha}$ initialized to zero.

The order of the numerator polynomials in (3-6) must be larger than that of the denominator polynomials so that the PR condition can be satisfied.

\subsection{Design Example}

The orders of the numerators and denominators of $H_{0}(z)$ and $H_{1}(z)$ are chosen to be 17 and 8 , respectively. The initial guess for the optimization is obtained from an 18-tap PR FIR filter banks. After constrained optimization, the IIR analysis filters $H_{0}(z)$ and $H_{1}(z)$ are factored by the factorization algorithm recently reported in [9]. This converts the nearly PR solution 
resulting from the optimization to a structurally PR system ( $H_{0}(z)$ and $\left.\hat{H}_{1}(z)\right)$ with slight decrease in stopband attenuation of $H_{1}(z)$. Details of this factorization will be reported elsewhere. The order of the numerator polynomial of $\hat{H}_{1}(z)$ is increased from 17 to 21 (Note that $H_{0}(z)$ remains unchanged). Fig. 2(a) shows respectively the magnitude responses of the optimized pseudo PR IIR analysis filter banks $H_{0}(z)$ and $H_{1}(z)$ (solid lines) and the factored structurally PR analysis highpass filter $\hat{H}_{1}(z)$ (dotted line). The group delays of $H_{0}(z)$ (solid line) and $\hat{H}_{1}(z)$ (dashed line) are shown in Fig. $2(\mathrm{~b})$, respectively. It can be seen from Fig. 2 (a) that the factored filter $\hat{H}_{1}(z)$ has slightly lower stopband attenuation than its pseudo PR counterpart. For fair comparison, the system delay (17 samples) and stopband cutoff frequencies are chosen to be identical to the example 1 in [2]. It is observed that they have comparable stopband attenuation of about $43 \mathrm{~dB}$. This demonstrates that the proposed method is able to suppress the bumping in $H_{1}(z)$ and control its stopband atsenuation.

\section{CAUSAL STABLE IIR WAVELET BASES}

\subsection{Theory and Design Procedure}

The theory of wavelets is closely related to that of multirate filter banks $[7,8]$. It can be shown that discrete dyadic wavelets can be obtained from two-channel PR filter banks with added regularity condition. For biothogonal dyadic wavelet bases, it had been proved that $H_{0}(z)$ and $G_{0}(z)$ should bave $K_{0}$ (or $\tilde{K}_{0}$ ) zeros at $z=-1$ (the $K$-regularity condition). This in turns require that $\tilde{N}_{0}(z)$ in (4-3) has $K_{0}$ zeros at $z=-1$. That is

$$
\tilde{N}_{0}(z)=\left(1+z^{-1}\right)^{K_{0}} Q_{0}(z),
$$

for some polynomial $Q_{0}(z)$ and integer $K_{0}>0$. In addition, $H_{1}(z)$ and $G_{1}(z)$ should also have at least one zero at $z=1$, that is

$$
\bar{N}_{1}(z)=\left(1-z^{-1}\right)^{K_{1}} Q_{1}(z)
$$

for some polynomial $Q_{1}(z)$ and integer $K_{1}>0$. By using the coefficients in $Q_{0}(z)$ and $Q_{1}(z)$ as variables, the regularity condition can be met exactly.

\subsection{Design Example}

A causal stable IIR wavelets basis with $K_{0}=\widetilde{K}_{0}=K_{1}=\bar{K}_{1}=1$ is designed by the algorithm of section 4 . The example in section 3 is chosen to be the initial guess. The orders of the numerator polynomials of $H_{0}(z)$ and $H_{1}(z)$ are 17 and 21 , respectively. Both of them have a denominator of order 8 . The system delay is 17 samples. Fig. 3(a) and Fig. 3(b) show respectively the magnitude responses of the analysis filters, and their scaling and wavelet functions. It can be seen that both the lowpass and highpass filters have zeros at $z=-1$ and $z=1$, respectively. It is also found that the waveform generated by iterating the IIR filter bank converge rapidly to the scaling and wavelets functions due to the effect of the poles in the denominators.

\section{TWO DIMENSIONAL NONSEPARABLE PR IIR FILTER BANKS}

In [2], a 1-D to 2-D mapping was proposed to transform the 1-D structural $P R$ IIR fitter bank to a 2-D diamond shape nonseparable PR filter bank. More specifically, the mapping replaces the 1-D transfer function $\beta(z)$ with the separable 2-D transfer function $\beta\left(z_{1}\right) \beta\left(z_{2}\right)$ and all I-D delay with the 2-D delay. The analysis and synthesis filters are given by,

$$
\begin{aligned}
& H_{0}\left(z_{0}, z_{1}\right)=\frac{z_{0}^{-2 N}+z_{01}^{-1} \beta\left(z_{0} z_{1}^{-1}\right) \beta\left(z_{1} z_{1}\right)}{2}, \\
& H_{1}\left(z_{0}, z_{1}\right)=-\beta\left(z_{1} z_{1}^{-1}\right) \beta\left(z_{0} z_{1}\right) H_{0}\left(z_{13}, z_{1}\right)+z_{10}^{-A N^{+}+1}, \\
& F_{0}\left(z_{0}, z_{1}\right)=-H_{1}\left(-z_{10},-z_{1}\right), F_{1}\left(z_{10}, z_{1}\right)=-H_{0}\left(-z_{01},-z_{1}\right) .(5-3)
\end{aligned}
$$

More properties of the mapping can be found in [2]. Careful examination reveals that it is also applicable to design 2-D filter bank from the optimized $\beta(z)$ obtained in section 2.2. Fig. 4(b) shows the contour plot of the magnitude response (in $\mathrm{dB}$ ) of the proposed 2-D.D IIR filter banks. For comparison purposes, the 2-D filters obtained by transforming the original FIR filters in section 2.2 are also included. It can be seen that the stopband attenuation and passband ripples of the proposed 2-D filters are better than that of the latter.

\section{CONCLUSION}

In this paper, two techniques for designing two-channel causal stable IIR PR filter banks are presented. The first method makes use of model reduction technique and constrained optimization to obtain a causal stable IIR filter bank from a given PR FIR prototype with better frequency characteristics. Using two dimensional nonseparable mapping, 2-D PR IIR filter bank can readily be obtained from their 1-D prototypes. Furthermore, a general PR condition and constrained optimization is proposed and filter banks with low system delay and flexible frequency characteristics can be designed. The technique can also be modified to design causal stable IIR dyadic wavelet bases with regularity conditions. The usefulness of the proposed design method is demonstrated by several design examples.

\section{REFERENCES}

[1] P. P. Vaidyanathan, Muttiraic systems and filter banks. Englewrood Cliffs, NJ: Prentice Hall, 1992.

[2] S. M. Phoong, C. W. Kim and P. P. Vaidyanathan, "A new class of two-channel biothogonal tilter banks and wavelet bases," IEEE Trans. SP, Vol.43, No. 3. pp.649-664, March 1995.

[3] M. Okuda, T. Fukuoka. M. Ikehara and S. Takahashi, " Design of causal IIR filter banks satisfying perfect 
reconstruction," Proc. IEEE ISCAS'97, Vol. 4, pp.24332436, June 1997.

[4] X. Zhang and T. Yoshikawa, "Design of causal IIR perfect reconstruction filter banks," Proc. IEEE ICASSP'98, Vol.3, pp.1429-1432, May 1998.

[5] T. Q. Nguyen and P. P. Vaidyanathan, "Two-channel perfect-reconstruction FIR QMF structures which yield linear-phase analysis and synthesis filters," IEEE Trans. Acoustics, Speech and Signal Processing, Vol. 37. No. 5 . pp. 676-690, May 1989.

[6] B. C. Moore, "Principal component analysis in linear system: Controllability, observability, and model reduction," IEEE Trans. Automat. Contr. Vol. AC-26, pp. 17-31, 1981.

[7] A. Cohen, I. Daubechies and J. Feauveau, "Biorthogonal bases of compactly supported wavelets," Comun. Pure Applied. Math. Vol. 45, pp. 485-560, 1992.

[8] S. C. Chan, Y. Luo, and K. L. Ho, "M-channel compactly supported biorthogonal cosine- modulated wavelets bases," IEEE Trans. Signal Processing Special Issue on Theory and Applications of Filter Banks and Wavelets, Vol. 46, No. 4, pp. 1142-1151, April 1998.

[9] S. C. Chan, "Factorization of M-Channel Biorthogonal Cosine-Modulated Filter Banks," internal report, Department of Electrical and Electronic Engineering, The University of Hong Kong, Dec. 1998.

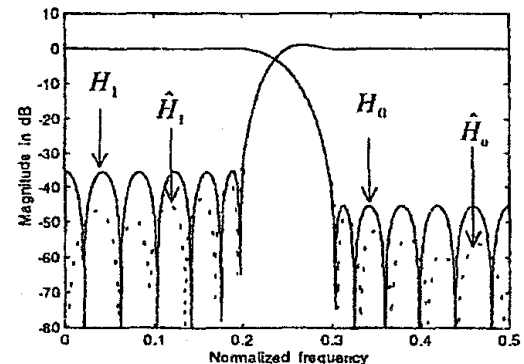

Fig. 1 Magnitude responses of analysis filter banks : solid lines - original FIR filters; dotted lines - optimized IIR filters

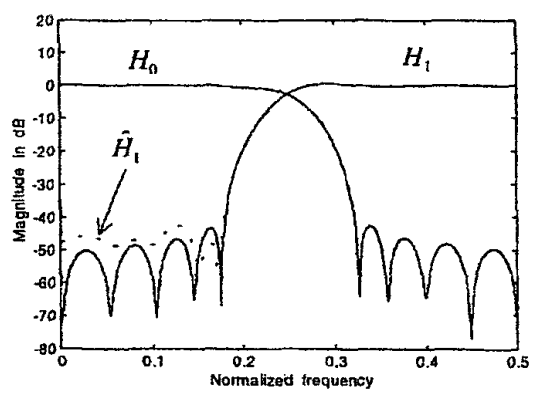

2(a)

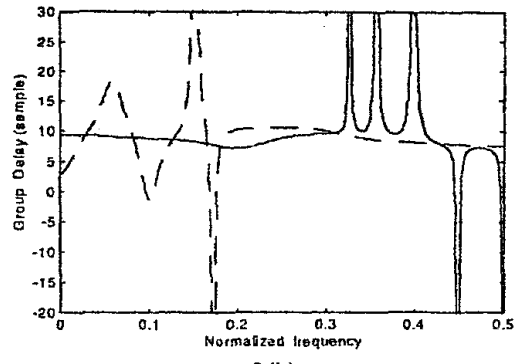

2 (b)

Fig. 2 (a) Magnitude responses of IIR PR analysis filter banks: optimized $H_{0}(z)$ and $H_{1}(z)$ (solid lines), factored $\hat{H}_{1}(z)$ (dotted line); (b) group delay of $H_{0}(z)$ (solid line), $\hat{H}_{1}(z)$ (dashed line).

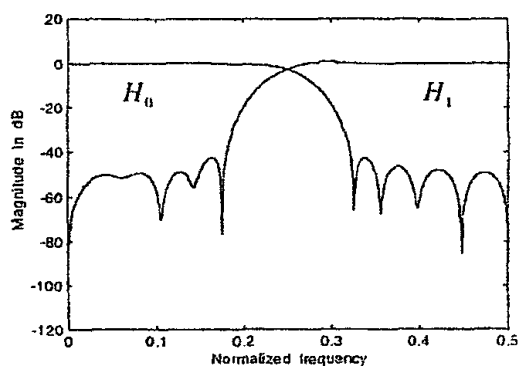

3(a)
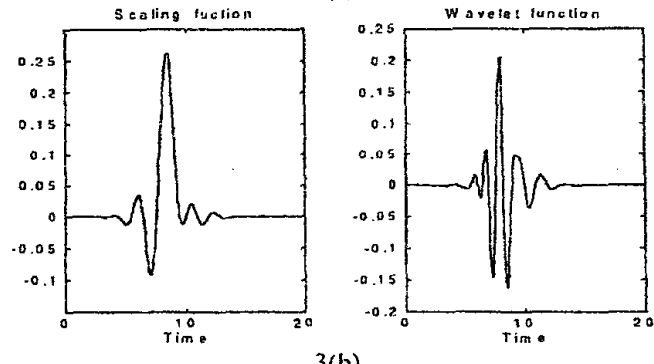

Fig. 3 Wavelet filter bank (a) magnitude responses of analysis filters; (b) analysis scaling function and wavelet function.

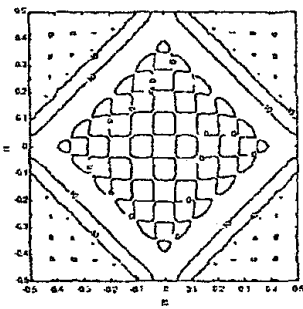

4(a)

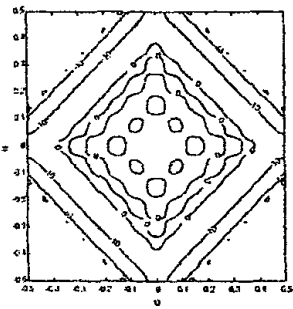

4(b)
Fig. 4 Contour plot of magnitude responses (dB) of 2-D PR analysis filter banks (a) lowpass FIR fîter proposed in [2]; (b) lowpass IIR filter proposed in this paper. The normalized frequency is $f_{i}=\omega_{i} / 2 \pi, i=0,1$. 